\title{
Patrimoines gastronomiques et développement local : les limites du modèle français de gastronomie élitiste
}

\section{Christian Barrère}

\section{(2) OpenEdition}

\section{Journals}

Édition électronique

URL : http://journals.openedition.org/tourisme/178

DOI : 10.4000/tourisme.178

ISSN : 2492-7503

Éditeur

Éditions touristiques européennes

\section{Édition imprimée}

Date de publication : 1 juin 2013

Pagination : 15-36

ISSN : 2109-5671

Référence électronique

Christian Barrère, «Patrimoines gastronomiques et développement local : les limites du modèle français de gastronomie élitiste », Mondes du Tourisme [En ligne], 7 | 2013, mis en ligne le 30 septembre 2015, consulté le 10 décembre 2020. URL : http://journals.openedition.org/tourisme/178 ; DOI : https://doi.org/10.4000/tourisme.178

\section{(c) $(1) \&$}

Mondes du tourisme est mis à disposition selon les termes de la licence Creative Commons Attribution - Pas d'Utilisation Commerciale - Pas de Modification 4.0 International. 


\title{
Patrimoines gastronomiques et
}

\section{développement local :}

\section{les limites du modèle français}

\section{de gastronomie élitiste}

\author{
CHRISTIAN B ARRÈ RE_ [christian,barrere@univ-reims.fr.] \\ Professeur de sciences économiques \\ Laboratoire Regards, Université de Reims Champagne-Ardenne
}

Résumé. Pour comprendre la diversité des effets sur le développement local de la gastronomie, nous partons d'un cadre d'analyse du développement gastronomique en liant les tendances de développement d'une gastronomie au modèle qui la structure et au patrimoine gastronomique qui l'englobe. Nous l'appliquons à la gastronomie française en étudiant son patrimoine gastronomique et ses effets d'entraînement sur les territoires. Nous relevons enfin leurs limites, compte tenu de l'épuisement relatif du modèle élitiste qui domine cette gastronomie.

Abstract. To understand the diversity of gastronomy - local development relation, the article proposes a framework which connects trends of gastronomic development, model of gastronomy and gastronomic heritage. We, then, use this framework to study the French gastronomic heritage and the relation between heritage and local development. Finally we specify the limits of this relation as the elitist model which dominates French gastronomy works out. 
$\mathbf{L}$ es liens observés entre développement de la gastronomie et développement local sont extrêmement variables. Dans certains cas, la gastronomie locale a pu être un facteur de développement, en particulier lorsqu'elle a su créer de véritables districts gastronomiques comme dans le cas de la région lyonnaise (Bonnard, 20l I) ou lorsqu'une "rénovation” gastronomique s'est appuyée sur des spécificités locales de ressources, voire les a développées comme dans le cas emblématique de Michel Bras (Etcheverria, 20I la et b ; Marcilhac, 20II ; Guichard-Anguis, 20II). En revanche, dans d'autres cas, hier mais aussi aujourd'hui, l'expansion du marché gastronomique se fait sans effets d'entraînement locaux particuliers (en dehors des effets habituels de dépenses de revenus liés à l'offre gastronomique), notamment en matière de promotion des produits locaux. Il en est ainsi pour une grande partie de la gastronomie “élitiste” (les “étoilés Michelin”), en France comme en Italie ou en Grande-Bretagne, même si des inflexions voient désormais le jour. Cette diversité, dans le temps et dans l'espace, des relations entre gastronomie et territoire s'observe aussi dans des domaines proches, comme en matière d'agrotourisme, d'œnotourisme et de promotion des produits agricoles et vitivinicoles de terroir (Gatelier, Delaplace et Pichery, 2012). De telles constatations suggèrent que divers types de liens puissent exister et qu'ils dépendent principalement du type même de déve- loppement de la gastronomie considérée. L'analyse de l'évolution des gastronomies montre en effet que ces développements sont loin de suivre une dynamique commune et qu'il est nécessaire de réintroduire leurs spécificités. Il en est ainsi quand on compare l'évolution des gastronomies fondées sur des districts gastronomiques et de celles qui ne le sont pas ou de celles qui sont fondées sur des patrimoines gastronomiques différents, comme la gastronomie française et la gastronomie italienne (Barrère, Buzio, Mariotti, Corsi et Borrione, 2012).

Il n'est alors pas possible de poser directement la question de la relation en général entre développement gastronomique et développement local sauf à assumer le risque de ne pouvoir dépasser les monographies : comment telle gastronomie et tel développement gastronomique ontils joué sur le développement de telle région ? Pour tenter de dépasser ces limites, il convient dans un premier temps de construire un cadre théorique suffisant pour analyser le développement gastronomique, voire en dresser des typologies, de façon à pouvoir, dans un second temps, lier formes de développement gastronomique et effets sur le développement des territoires. Le texte ciaprès s'inscrit dans le premier type de tâche, l'appréhension des types de développement gastronomique, même s'il propose une esquisse (et une esquisse seulement) de l'effet de la gastronomie française sur les territoires. Pour cela, il propose, dans un premier temps, les bases d'un cadre d'analyse du développement gastronomique en liant les tendances de développement d'une gastronomie au modèle qui la structure et au patrimoine gastronomique qui l'englobe. Il enrichit ensuite ce cadre en l'appliquant, dans un second temps, au développement de la gastronomie française par l'étude du patrimoine gastronomique français dans ses diverses composantes, élitiste et non élitiste. Il poursuit en proposant un début d'analyse des effets d'entraînement sur les territoires du système gastronomique français et de son patrimoine. Dans un quatrième temps, il montre enfin leurs limites, compte tenu de l'épuisement relatif $\mathrm{du}$ modèle élitiste qui les domine, et conclut en soulevant la question de la relève possible par les composantes non élitistes, régionales et locales, du patrimoine gastronomique français, susceptibles de poser en termes nouveaux le problème de la relation développement gastronomique - développement local.

\section{UN CADRE ANALYTIQUE POUR PENSER \\ LE DÉVELOPPEMENT GASTRONOMIQUE}

La constatation de la régularité dans le temps et l'espace de pratiques alimentaires définies et particulières a conduit Lévi-Strauss à analyser le caractère culturel de ces pratiques qui pouvaient sembler "naturelles" en reliant "naturellement" des individus ou des groupes aux ressources disponibles localement. Ce travail 
fondateur a ouvert la voie à l'analyse de la cuisine comme "fait social total", selon la formule de Mauss. Fischler (1990) identifie des "cultures culinaires", ensembles plus ou moins cohérents et durables de principes de choix, de combinaison et de préparation des aliments pour la nutrition. Il définit en outre des "systèmes culinaires", ensembles de règles culturelles qui organisent ce qu'on peut et ne peut pas manger, les formes sous lesquelles les aliments sont préparés et consommés, les personnes et groupes auxquels sont destinés les aliments... Le système culinaire fixe également les règles d'association des aliments (qui prendront, dans la cuisine classique, la forme de recettes) permettant de créer de la variété à partir de mêmes aliments de base.

En montrant la spécificité du modèle français des trois repas structurés, Poulain (2002) introduit la notion de "modèle alimentaire", réservant les termes de cuisine et de système culinaire aux règles de préparation des éléments: "Les modèles alimentaires sont des ensembles sociotechniques et symboliques qui articulent un groupe humain à son milieu, fondent son identité et assurent la mise en place de processus de différenciation sociale interne. Ils sont un corps de connaissances technologiques, accumulées de génération en génération, permettant de sélectionner des ressources dans un espace naturel, de les préparer pour en faire des aliments, puis des plats, et de les consommer. Mais ils sont en même temps des systèmes de codes symboliques qui mettent en scène les valeurs d'un groupe bumain participant à la construction des identités culturelles et aux processus de personnalisation" (Poulain, 2002, p. 25).

La gastronomie ne s'identifie pas à la cuisine ou à l'alimentation. Elle apparaît comme ensemble autonome en se détachant de l'alimentation quand, à telle époque, dans tel lieu et pour tel groupe social, le souci du plaisir l'emporte sur le souci nutritionnel et devient recherché en tant que tel, donc lorsque la cuisine sort du registre de la nécessité pour entrer dans celui du plaisir ${ }^{(1)}$. Elle est, elle aussi, structurée comme en témoigne encore récemment la décision de l'Unesco de distinguer "le repas gastronomique des Français" et d'en faire un élément spécifique du patrimoine mondial. Elle l'est d'autant plus que la gastronomie devient une activité professionnelle qui, de ce fait, a besoin de codifier ses règles et d'en assurer l'enseignement et la transmission entre générations.

Chaque cuisine, culture culinaire ou modèle alimentaire n'engendre pas de gastronomie correspondante. Les gastronomies émergent quand certaines conditions sont réunies, culturelles et sociales. Dans le monde oriental, elles sont liées à la puissance des empires et à l'instauration de sociétés de cour ; dans le monde occidental, depuis la Renaissance, se développent également des gastronomies, dont la partie émergente est, elle aussi, liée aux sociétés de cour. On peut alors s'efforcer d'iden- tifier des modèles gastronomiques, entendus comme ensembles, plus ou moins cohérents, de principes, de normes et de règles, s'appliquant au statut de la gastronomie dans la société, à ses règles de composition, de fonctionnement et de transmission-renouvellement. Formellement, nous pouvons distinguer des principes structurels (quels produits sont utilisés, quelle valeur leur est attribuée, qui consomme de la gastronomie, à quelles occasions, sous quelles formes, qui produit la gastronomie, quelle est la configuration du système d'acteurs...) ; des principes de fonctionnement, assurant la régulation de l'ensemble (par exemple, comment sont formés et recrutés les chefs dans les systèmes fondés sur leur hégémonie ou comment est assurée, dans les régimes marchands, l'adaptation entre offre et demande) ; et des principes de développement (la transmission entre générations, le culte du patrimoine, l'incitation ou non à l'innovation...), qui débouchent sur des sentiers d'évolution (course à la sophistication ou à la créativité ou, au contraire, conservatisme culinaire ; flexibilité et degré d'ouverture aux influences extérieures ; capacité à s'exporter hors de son territoire d'origine...) et seront à la base du développement local des gastronomies.

Une gastronomie peut s'organiser autour de plusieurs modèles. Ainsi dans le cas français distingueronsnous un modèle de gastronomie élitiste et un modèle, très différent, de gastronomie populaire. Dans la 


\section{Figure | • Le système gastronomique}

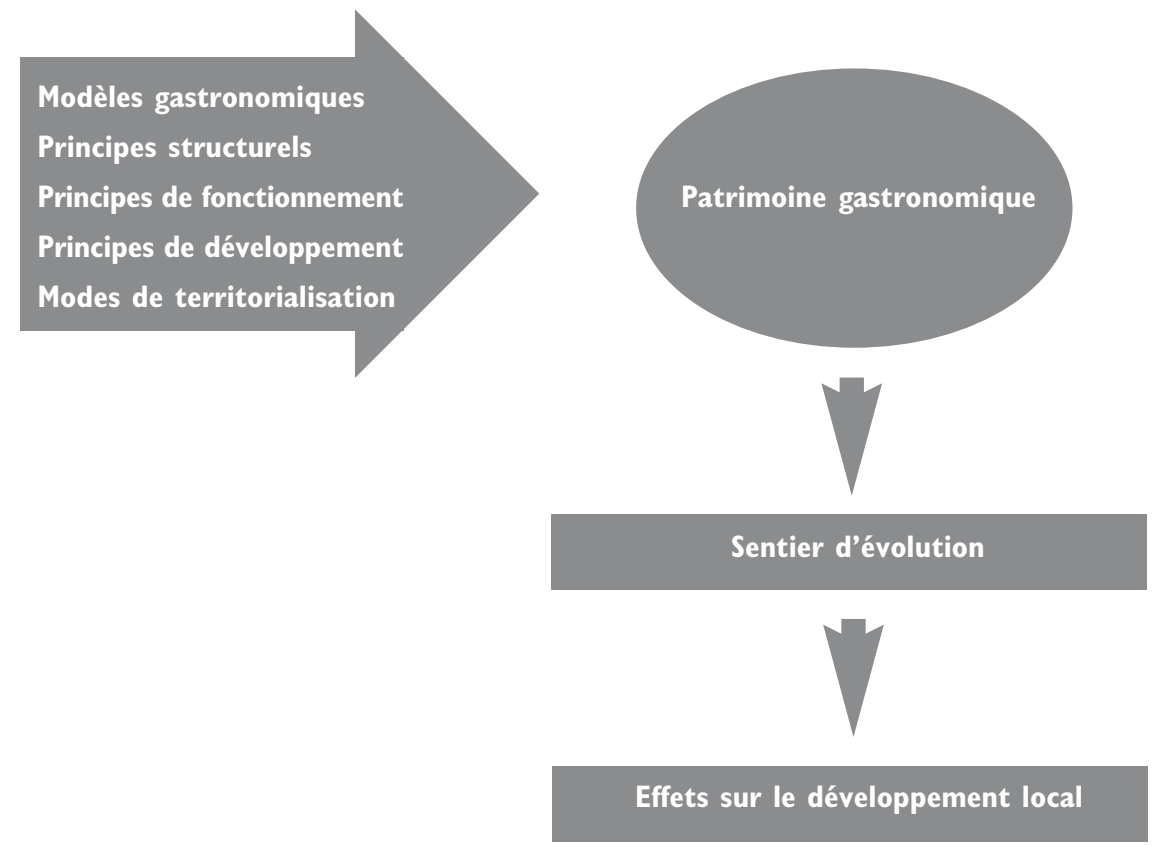

Modèles gastronomiques

Principes structurels

Principes de fonctionnement

Principes de développement Modes de territorialisation mesure où s'établissent des relations entre ces modèles (relations de concurrence, de complémentarité, de domination...), ils participent d'un système gastronomique spécifique ( $c f$. figure 1 ). Le système gastronomique français s'organise autour de la relation hiérarchisée gastronomie élitiste-gastronomie populaire. De tels systèmes présentent des éléments de cohérence, qui permettront par exemple de distinguer au niveau mondial une gastronomie française, mais cette cohérence est loin d'être parfaite dans la mesure où ils peuvent résulter de fondements hétérogènes (la cuisine de la cour et la cuisine paysanne), où ceux-ci s'influencent les uns les autres, et rencontrent d'autres sys- tèmes, d'autres cultures culinaires et gastronomiques.

La reproduction dans le temps des modèles et du système aboutit à la constitution de patrimoines gastronomiques. Les patrimoines gastronomiques (le patrimoine français comme le patrimoine chinois) cristallisent les principes structurels de fonctionnement des systèmes gastronomiques et les transmettent à travers le temps. Ils évoluent, intègrent de nouveaux éléments, en perdent d'autres, en fonction des principes de développement des modèles de base qui les constituent (ainsi le patrimoine français de la gastronomie aristocratique puis élitiste accumule-t-il de plus en plus de recettes dans la mesure où ce modèle pousse à la recherche de nouvelles recettes et à l'innovation technologique), de leurs formes particulières de composition dans un système gastronomique et de leur confrontation à d'autres systèmes (la cuisine française rencontre les cuisines étrangères).

Nous entendrons ces patrimoines comme des ensembles, attachés à des groupes, situés dans le temps et l'espace, exprimant des composantes de leur spécificité et donc de leur identité, ensembles historiquement institués d'avoirs, construits et non construits, transmis par le passé (Barrère, Barthélemy, Nieddu et Vivien, 2004, p. I 16), avoirs qui incluent des actifs matériels (des équipements, des locaux...), des actifs immatériels 
(des savoir-faire, des recettes, des normes, des réputations...) et des institutions (les chambres syndicales, les organismes de formation...). Ils résultent d'une construction et d'une sélection sociale de leurs composantes par des communautés et des individus déterminés (Louis XIV et la reine Victoria joueront un rôle essentiel dans la consécration d'un modèle gastronomique français ou britannique), dans des contextes donnés (ainsi de la domination du puritanisme dans certains pays ou de la philosophie libertine dans d'autres). Ils ont une dimension spatiale par la relation entre patrimoines et communautés, sont liés à des cultures localisées, la dimension locale pouvant fortement varier (dans le cas français, le modèle élitiste impose une dimension nationale tandis que subsistent des patrimoines régionaux ou locaux). Ils se reproduisent par un processus de transmission culturelle, par exemple via la conservation des traditions artisanales et la transmission des savoir-faire professionnels ou par une transmission mèrefille au sein des familles. Cependant, comme les systèmes gastronomiques dont ils proviennent, ces patrimoines, soumis à la rencontre d'autres patrimoines et d'autres cultures, dont des cultures culinaires, ne constituent pas des ensembles totalement cohérents, même si certaines caractéristiques fortes les marquent, et ils ne se reproduisent pas à l'identique. Ils s'enrichissent (nouvelles recettes, nouvelles techniques) ou s'appauvrissent en se figeant et en étant incapables de s'adapter aux évolutions sociales et culturelles; ils contribuent, selon les cas, à reproduire le ou les modèles gastronomiques qui sont à leur origine ou, au contraire, s'épuisent progressivement, face aux mutations, selon qu'ils facilitent ou "corsètent" les adaptations nécessaires en réponse à l'évolution de données socio-économiques plus larges (globalisation des économies, émergence de nouveaux pays et de nouveaux groupes sociaux à fort pouvoir d'achat, changements dans les valeurs...).

\section{CONSTITUTION}

\section{ET STRUCTURE DES MODÈLES} ET DU PATRIMOINE

GASTRONOMIQUES :

\section{LE CAS DE LA FRANCE}

Nous nous intéressons au cas de la France, d'une part parce que c'est celui sur lequel existent les études les plus approfondies, d'autre part parce que le patrimoine gastronomique français a eu un rôle international déterminant, de sorte que son avenir est au cœur des mutations actuelles de la gastronomie mondiale. Le système gastronomique français s'est historiquement constitué autour de deux modèles gastronomiques très différents, qu'il a rapprochés et partiellement unifiés, et a produit un très riche patrimoine gastronomique qui s'est largement diffusé à travers le monde.

\section{Deux modèles gastronomiques}

Les apports des historiens et sociologues de la gastronomie fran- çaise permettent d'avancer une caractérisation du système gastronomique français à partir de l'existence d'un patrimoine gastronomique français, rassemblant des éléments appartenant à deux modèles gastronomiques assez différents mais qui, vu leur proximité de base spatiale, ont réalisé des échanges et des hybridations. Un modèle élitiste "national" issu de la culture de cour, plutôt dominant, a coexisté pendant des siècles avec un modèle gastronomique "régional" et "populaire", du fait du processus historique de développement en France de la gastronomie.

La gastronomie française a, en effet, deux sources essentielles. D'un côté, les cuisines locales ont évolué vers des prestations gastronomiques. Sur la base de produits locaux (truffes, poissons, champignons, fromages, vins par exemple), d'une élaboration et d'une sélection locales (confits, garbures, bouillabaisse, cassoulets, tripes, charcuteries...) ont été définies des recettes locales, des plats locaux ou régionaux, pour la cuisine ordinaire mais aussi pour les fêtes et cérémonies (repas de mariage, de communion, fêtes religieuses...). Selon l'importance et la variété des produits locaux plusieurs cultures culinaires émergent (cuisine lyonnaise, alsacienne, du SudOuest...), avec un fort ancrage territorial. Elles privilégient certains produits, définissent des modes de préparation (le confit, les salaisons ou le fumage du saumon), sélectionnent des plats emblématiques (le cassoulet, la bouillabaisse), nor- 
malisent des recettes locales. Cette gastronomie populaire, qui a existé dans nombre de pays européens (Breughel peint les fêtes paysannes qui exhibent une pléthore de mets), n'est pas marginale, même si elle est limitée à des occasions et des périodes de temps définies. Les tableaux de Bosch illustrant le jugement dernier comprennent toujours la figure du gourmand, promis à l'enfer, figure que l'on retrouve sur les fresques des églises et monastères du Moyen Âge, témoignant que la "gloutonnerie" n'était pas exceptionnelle et n'était pas réservée aux grands.

D'un autre côté, la société de cour, les cours régionales puis la cour du Roi, ont fait naître puis consacré une cuisine aristocratique dont le but est d'affirmer la puissance des souverains, vis-à-vis des humbles d'un côté et des autres puissants et concurrents de l'autre. La concurrence entre pouvoirs locaux puis le besoin de consolider la domination du pouvoir royal enclenchent un processus de surenchère qui tend à caractériser de plus en plus la cuisine aristocratique par la sophistication et l'étalage de la richesse. La société du grand siècle de Louis XIV jouera un rôle exceptionnel dans la construction, la normalisation, la légitimation et l'exportation de ce modèle français à travers l'Europe. Celui-ci constitue la base d'un patrimoine gastronomique durable (type de plats, ordonnancement du menu, recettes, type de service, modes de présentation, ornementation de la table, vaisselle, verrerie...) qui évo- luera au xixe siècle avec le passage d'un modèle proprement aristocratique à un modèle élitiste, quand les mutations sociales mettront au premier plan de nouveaux groupes sociaux.

La bourgeoisie hésitera entre cuisine populaire et aristocratique et intégrera progressivement pour se distinguer du reste du peuple une version "euphémisée" de la cuisine aristocratique.

\section{L'évolution vers un modèle élitiste qui domine le système gastronomique français}

Au Moyen Âge, le banquet aristocratique, dans tous les pays, était caractérisé par l'excès. Profusion de plats, de produits rares et riches, gaspillage... Avec la cuisine de cour, le chef devient élément de paraître pour les grands, qui cherchent à les attirer. Pour les épater, le "grand cuisinier" fait appel à sa créativité : nouvelles recettes, nouvelles sauces, nouvelles présentations... Alors que la gastronomie populaire est plutôt conservatrice, la cuisine aristocratique $\mathrm{du}$ Grand Siècle glorifie l'invention. Le chef passe du statut d'artisan à celui d'artiste, la cuisine n'est plus une activité matérielle mais une œuvre intellectualisée, esthétisée et formalisée (Parkhurst Ferguson, 2004).

La chute de l'aristocratie avec la Révolution française s'accompagne d'une marchandisation de la cuisine ; les anciens cuisiniers des cercles aristocratiques se reconvertissent en ouvrant des restaurants (il y avait cinquante restaurants en 1789 mais trois mille en 1815). La plupart d'entre eux développèrent une version "euphémisée" de la cuisine aristocratique pour la bourgeoisie moyenne et supérieure, qui fut le vrai vainqueur de la période révolutionnaire, de l'Empire et de la Restauration. Ayant le pouvoir économique et une bonne part du pouvoir politique, elle constitua le noyau des élites dominantes. La cuisine bourgeoise est "euphémisée" car une partie de la bourgeoisie, y compris supérieure, travaille de sorte que la "grande" cuisine reste limitée à des occasions définies, les réceptions, les banquets... à l'intérieur de la "bonne société".

Brillat-Savarin (1755-1826) et Antonin Carême (1783-1833) furent les principaux acteurs de cette normalisation. Ils créèrent un système cohérent de sauces, soupes, pâtisseries, accompagnements de légumes (Parkhurst Ferguson, 2004, p. 32), rompant avec l'extravagance de la cuisine de cour mais trouvant leur inspiration dans ce patrimoine (Brillat-Savarin, I 848 [paru en 1826]). De même sélectionnèrent-ils au sein des cuisines populaires et régionales les plats et recettes méritant d'être intégrés à la "cuisine française légitime", en en excluant d'autres, considérés comme "vulgaires" ou "locaux". Ils contribuèrent ainsi à unifier dans un patrimoine unique, celui de la cuisine française et plus particulièrement de sa gastronomie, les éléments, plus ou moins hétérogènes, provenant des deux modèles précités.

L'association entre base aristocratique et version bourgeoise de la gastronomie française est demeurée 
le cœur du système gastronomique français, même lorsque les cours impériales de Napoléon I ${ }^{\text {er }}$ et de Napoléon III développèrent, de nouveau, luxe et sophistication. Un tel système est à dominante élitiste, le modèle élitiste apparaissant comme plus valorisé que le modèle populaire et constituant le sommet de la pyramide culinaire. Il s'inscrit dans un paradigme spécifique, élitiste, du goût. L'étude de l'évolution de la mode (Spencer, 1854 ; Veblen, I899; Simmel, 1904 ; Lipovetsky, 1987 ; Barrère et Santagata, 2005) et de la gastronomie (Parkhurst Ferguson, 2004) a montré que le statut social du goût et du luxe est régi par différents paradigmes. Ces paradigmes organisent les questions et réponses relatives au statut et à la définition du goût et des biens de goût, pour une société donnée, à un moment donné. Comment définir le bon goût et le mauvais goût? Qui les définit ( $c f$. l'image des “arbitres des élégances”) ? Quels biens ou types de biens appartiennent au domaine du luxe? Quand et comment utiliser les biens de luxe ? Qu'est-ce qui est ordinaire et qu'est-ce qui est extraordinaire, est cuisine ou est gastronomie ?

Le paradigme aristocratique de la société concevait la société comme un ensemble de deux sous-ensembles, deux classes : la noblesse et le peuple. Le paradigme du goût et du luxe aristocratique tournait autour de l'idée que le bon goût est spécifique aux grands et ainsi que les biens de luxe devaient leur être réservés ; le peuple ne saurait les apprécier, ce serait "donner de la confiture aux cochons". Le vocabulaire du luxe aristocratique exprime encore cette relation : la haute couture, la grande cuisine, les grands vins. Les biens de goût aristocratiques et les biens de goût populaires étaient totalement disjoints et différents, les biens de luxe et les biens ordinaires radicalement séparés, les consommateurs s'“auto-sélectionnaient”. Ce paradigme a historiquement évolué en un paradigme élitiste quand l'ancienne noblesse s'est vue supplantée par la nouvelle bourgeoise d'affaires, les élites intellectuelles et artistiques. L'évolution du modèle gastronomique participe de ce mouvement.

Curnonsky (1872-1956), en écrivant l'énorme somme que constitue La France gastronomique (28 volumes), a définitivement codifié la "grande cuisine" et la "cuisine bourgeoise”. Le Second Empire fut une période d'expansion de nouveaux groupes sociaux appartenant à l'élite, la bourgeoisie industrielle et financière, composée de banquiers, d'industriels, de grands commerçants et d'intermédiaires. Ils étaient riches, cherchaient à se fondre dans la vieille aristocratie, s'intéressaient aux arts et voulaient être distingués du peuple, ainsi que Veblen (1899) les analysera. Un modèle économique du luxe élitiste apparaît alors et renforce la cohérence du modèle de gastronomie élitiste.

\section{L'organisation du système gastronomique français}

Si la cuisine bourgeoise régnait sur l'ensemble du territoire français, la gastronomie aristocratique, née dans les palais de la cour, était principalement concentrée à Paris. La gastronomie élitiste a maintenu cette localisation qui correspondait aussi à celle des élites françaises, et de plus permettait d'attirer de nombreux touristes étrangers.

La gastronomie élitiste, comme c'était déjà le cas de la cuisine de cour (on se souvient du suicide de Vatel, cuisinier du prince de Condé, en raison du retard de la "marée" venue des côtes normandes), draine vers Paris tous les produits de qualité disponibles sur le territoire français, aidée en cela par la centralisation jacobine des transports ( $c f$. aujourd'hui le rôle du marché national de Rungis où l'on peut trouver tous les produits frais possibles, y compris venant de l'étranger). La chaîne de production est ainsi extrêmement longue. Les produits utilisés doivent être rares, "nobles" (langouste, homard, foie gras, ris de veau, caviar, huîtres, asperges, turbot...), de très grande qualité. Ils sont aussi extrêmement variés du fait de la grande diversité des terroirs et climats propre à la France. Ils sont travaillés par des personnes hautement qualifiées, dans des processus de préparation longs et complexes, en recourant à des techniques et à des appareillages sophistiqués. Le travail est organisé de façon rigoureuse, via une division des tâches précise : la "brigade" avec ses divisions, "sauciers", "légumiers", pâtissiers... La cuisine est une activité professionnelle, respectant des normes et routines déterminées, impliquant formation spécifique et 
longue, souvent à travers le passage dans différents établissements, à la manière du "Tour de France" des compagnons. Le chef est un créateur, avec un statut proche de celui du couturier créateur de la haute couture tout en étant généralement issu d'un apprentissage complet de la hiérarchie culinaire, ce dont il se montre fier en arborant sur sa veste l'insigne de "meilleur ouvrier de France".

Le grand restaurant constitue le cœur du modèle tout en étant à la pointe de la pyramide gastronomique et occupant sa niche élitiste, diffusant ses innovations vers les segments inférieurs dans une logique top-down. Ses recettes manifestent de la sophistication, de la créativité, sont fortement consommatrices en temps. La vaisselle et la verrerie, les nappes, le décor, le service exhibent leur luxe. Le restaurant offre une profusion de plats, avec un choix large, de multiples accessoires (une cave riche, des cigares, des alcools...). Coûts et prix sont élevés ou très élevés. Le modèle est un modèle d'offre, le cuisinier ne répondant pas à une demande préalable de la clientèle mais définissant le produit offert, qui cherche alors sa clientèle.

La critique gastronomique légitime ce modèle en promouvant les grands chefs et en exigeant d'eux un cadre luxueux. Les conventions culinaires, comme la "nouvelle cuisine" dans les années 1960 ou la "cuisine créative" aujourd'hui, dévalorisent à l'inverse la cuisine bourgeoise et populaire, régionale, "trop lourde", tout juste bonne à être "revisitée" et "sophistiquée" par les grands chefs. La gastronomie élitiste se distingue clairement de la gastronomie populaire. Le "cuisinier", le chef, s'oppose à la "cuisinière", à la "ménagère", comme le "grand couturier" à la "couturière". $\mathrm{Au}$ lieu de privilégier le produit la gastronomie élitiste esthétise la cuisine et donne de l'importance à l'apprêt et à la présentation du plat. Elle développe l'intellectualisation et la "scientifisation" de la cuisine (Parkhurst Ferguson, 2004). Loin de se contenter de recourir aux recettes et routines traditionnelles, les chefs cherchent à comprendre les "lois" de la cuisine, les processus de transformation des inputs pour les mâ̂triser et les sophistiquer, les mécanismes du goût.

\section{La reproduction du système par le patrimoine}

La gastronomie élitiste puise dans le patrimoine français de la cuisine qui intègre différents types d'éléments, issus de la cristallisation, à travers les siècles, de la gastronomie aristocratique et élitiste mais aussi des gastronomies locales et populaires ; elle s'en sert de façon spécifique, différemment des cuisines populaires et locales, en fonction de ses principes structurels et de fonctionnement. Plus précisément, le patrimoine de la cuisine française rassemble des éléments en partie hétérogènes : patrimoines culinaires et patrimoines gastronomiques, patrimoine élitiste et patrimoines locaux (la cuisine niçoise) ou régionaux (la cuisine du Sud-Ouest). Il constitue cependant une certaine unité, par les échanges qui ont pu intervenir entre ses différents composants et par la culture commune dans laquelle ils s'inscrivent. Comme nous l'avons dit, rien n'interdit au grand chef de s'inspirer de recettes ancestrales et locales de même que la ménagère ou le cadre "bobo" peut tenter de réaliser les recettes sophistiquées des grands chefs diffusées par la presse ou sur internet. En même temps, il reflète la domination symbolique de sa composante élitiste.

Ce patrimoine est d'abord un patrimoine de savoir-faire et de création, savoir-faire des cuisiniers professionnels et savoir-faire créatif, source d'avantages comparatifs susceptibles d'attirer une clientèle nationale et internationale. Il l'est via des effets de stock et de mémoire : stocks de recettes et de techniques, que l'on peut utiliser mais aussi "revisiter" ; mémoire des grands cuisiniers d'antan, de leurs pratiques et de leurs discours. Il l'est aussi via des effets d'expérience : les "anciens" transmettent aux plus jeunes via l'apprentissage, le travail en commun ; les participants à la création culinaire peuvent élever leur créativité du fait de leur insertion dans une communauté culturelle qui a réfléchi sur la gastronomie, en connaît l'histoire. Il l'est encore par des effets de district culturel : le patrimoine gastronomique s'inscrit dans une communauté culturelle s'exprimant par un "style" français du luxe ou un "goût" français et bénéficie des créations réalisées dans d'autres domaines. C'est aussi un patrimoine 
institutionnel, avec des institutions définies, les systèmes d'apprentissage, d'échange entre professionnels, de relations avec la critique gastronomique...

Le patrimoine ne participe pas seulement de la production de biens mais aussi de la production de leurs débouchés. La consolidation et l'extension des débouchés de la gastronomie française se sont appuyées sur un patrimoine de préférences et de goûts, l'existence d'un fonds commun partagé par une communauté qui a appris à lire de façon homogène les divers signes, qui met le même système de signifiants derrière le même système de signes et assure l'homogénéité des représentations, de la relation signifiantsignifié, donc des "goûts" à la fois des offreurs et des demandeurs. Ce patrimoine facilite ainsi, sur un marché de "produits signes", la rencontre entre offre et demande, rend la gastronomie lisible donc la justifie et la légitime au nom du lien au passé et à l'histoire. Le patrimoine crédibilise la création qui s'y réfère et en élève la réputation.

\section{SENTIER DE DÉVELOPPEMENT ET EFFETS D’ENTRAÎNEMENT DU PATRIMOINE}

\section{À DOMINANTE ÉLITISTE}

Le patrimoine à dominante élitiste contribue à la reproduction de la cuisine élitiste le long d'un sentier de développement précis. Son rapport particulier an territoire est alors à la source d'effets particuliers sur le développement territorial.

\section{Le sentier de développement}

Le patrimoine gastronomique a deux effets importants. En premier lieu, il transmet, en tant que patrimoine territorial partagé, celui de la France, une culture commune d'autant plus forte que les principes de la cuisine et de la gastronomie ont été définis, codifiés, articulés et normalisés (nous avons mentionné le rôle de Carême ou de Curnonsky), puis diffusés (les livres de recettes mais aussi les "manuels d'éducation ménagère”). Il est alors source d'économies de coordination : langage commun, confiance mutuelle, partage de connaissances tacites, circulation aisée de l'information, formes de coopération malgré la concurrence, possibilité dans certaines conditions de favoriser la constitution de districts gastronomiques. En second lieu, il développe des effets d'idiosyncrasie, en permettant à la production française de bénéficier de spécificités d'actifs, engendrées tout au long de l'histoire et sources d'avantages comparatifs décisifs.

Il tend à établir un cercle vertueux créativité-patrimoine :

- l'histoire de la création culinaire et le culte du créateur, le grand cuisinier, sont des facteurs incitant à la créativité, en la valorisant ; - les effets de stock, de mémoire et d'expérience facilitent l'apprentissage et la transmission de la créativité, notamment entre générations ; - la patrimonialisation de la cuisine française, et particulièrement de sa gastronomie, la rapproche de l'art et de la création et l'insère dans le patrimoine intellectuel, artistique et culturel français. Cela facilite la transmission des inventions entre disciplines (ainsi, hier le recours à une présentation architecturale des desserts ou aujourd'hui l'invention du design culinaire) et crée une émulation pour la créativité.

La concurrence entre offreurs passe avant tout par la course à la créativité, le renouvellement de la carte et l'investissement dans le cadre du restaurant. Experts et guides, en premier lieu le Guide rouge, justifient ce modèle et disent qui appartient à quel segment, de la niche élitiste des restaurants étoilés et prestigieux aux restaurants standards, en passant par les différents segments définis par des prix et une qualité décroissants mais se référant à des mêmes principes culinaires. Ils contribuent aussi à développer le modèle élitiste et la segmentation correspondante dans les "nouveaux" pays et villes gastronomiques (Espagne, Italie, Japon, Londres, New York, ...), où la consommation de gastronomie s'étend rapidement. La course à la créativité, au luxe et aux étoiles peut être illustrée par l'exemple d'Alain Ducasse qui, quand il fut engagé comme chef du Louis XV, à Monte-Carlo, avait d'après son contrat un an pour obtenir la troisième étoile Michelin, faute de quoi celui-ci était automatiquement rompu.

La consommation de masse de gastronomie, sa marchandisation et sa démocratisation ont continué, au moins jusqu'à la fin du siècle dernier, à se faire dans le cadre du 
modèle élitiste. Le point de référence est resté le grand restaurant et les caractéristiques typiques du vieux modèle aristocratique : un chef célèbre, des produits extra-ordinaires (truffes, caviar, langouste...), des plats sophistiqués, un service de qualité, un décor luxueux et, in fine, une cuisine coûteuse. Ainsi, l'intérêt croissant pour la gastronomie ne s'est pas seulement traduit par une inflation du discours gastronomique. La fréquentation des restaurants gastronomiques s'est fortement élevée bien que les prix pratiqués, absolus et relatifs, aient continué de grimper et soient parfois devenus surréalistes.

Le nombre de restaurants gastronomiques présents dans le Guide Michelin entre 1934 et 2010 et situés à Paris a fortement augmenté au cours de cette période (Barrère, Bonnard et Chossat, 2010) : 193 en 1934, 324 en 1950 et 493 en 2010 ; soit une hausse de $155 \%$ de 1934 à 2010 et de $52 \%$ de 1950 à 2010. Le champ gastronomique s'est étendu en dépit de la hausse, continue et forte, des prix. Les prix moyens des menus et des repas à la carte, observés en euros constants, ont respectivement grimpé de $+245 \%$ et de $+208 \%$ entre 1950 et 2010. Malgré la croissance des prix, la demande de gastronomie n'est plus une demande limitée à un marché étroit mais est devenue une demande de masse.

En son sein, la niche élitiste concernant les prestations de très haut niveau s'est elle aussi étendue bien que ce soient les prix des pres- tations "les plus sophistiquées" qui augmentent relativement le plus sur la période (les prix à la carte ont plus augmenté que les prix des menus et les prix les plus élevés au départ davantage que les moins élevés), mouvement qui s'accélère encore après 2000. Cette demande émane de personnes financièrement très riches et a donc une élasticitéprix très faible. Comme elles sont présentes dans un nombre croissant de pays et peuvent voyager partout dans le monde, elles sont suffisantes pour alimenter la demande des restaurants superstars (les deux ou trois étoiles Michelin, avec une addition de plus de 200 euros par personne, hors boisson).

Le deuxième type de demande de haute gastronomie, la demande de masse de gastronomie de luxe, s'est lui aussi fortement accru. Ainsi, dans le secteur de la gastronomie comme dans le secteur du luxe, une demande régulière et occasionnelle a donné naissance à une demande de masse (les restaurants sélectionnés par le Guide Michelin en France, qui constituent environ $8 \%$ seulement du nombre de restaurants, peuvent être définis comme "gastronomiques" et représentent néanmoins plus de 8000 restaurants), demande qui s'adresse à une offre qui est restée organisée sur le modèle du grand restaurant et de la cuisine intellectualisée, formalisée et esthétisée, du modèle élitiste. La croissance de cette demande de haute gastronomie est également attestée par l'observation des comportements des groupes financiers et groupes du luxe qui ont investi dans les restaurants et hôtels. Les palaces parisiens ont recruté des chefs de haut niveau pour gagner des étoiles au Michelin, asseoir leur réputation, accroître ou préserver leur clientèle de touristes.

Le modèle gastronomique élitiste a également attiré les touristes étrangers qui, par la suite, ont constitué, dans leurs pays, une source de demande pour de nouveaux offreurs locaux s'inspirant de cette gastronomie. Le modèle élitiste français s'est ainsi exporté comme s'était déjà exporté dans les cours européennes le modèle aristocratique du Grand Siècle. Cela était d'autant plus faisable que, comme le fait très justement remarquer Parkhurst Ferguson, la cuisine de Carême n'était plus un ensemble de connaissances empiriques mais un savoir scientifique fondé sur un apprentissage technique et professionnel, un système cohérent de principes et de techniques d'application "Carême's French cuisine is not tied to or rooted in a particular place" [La cuisine française de Carême n'est pas liée à ni ne prend racine dans un lieu particulier] (Parkhurst Ferguson, 2004, p. 7I). En outre, la diminution des coûts de transport, alors que les prix exigibles étaient très élevés, permettait de se procurer l'essentiel des produits rares et nobles, typiques de la cuisine élitiste. Au pire il était possible de remplacer certains produits français par des produits locaux.

Ainsi en Espagne, en Italie, dans les pays nordiques et jusqu'au 
Royaume-Uni, pourtant jadis réputé pour la médiocrité de sa nourriture et l'absence de réelle gastronomie, se sont développés des restaurants gastronomiques tout à fait comparables aux restaurants français. L'extension du Guide Michelin à ces nouvelles contrées gastronomiques atteste et de la présence de restaurants de grande qualité et de leur insertion dans le modèle de gastronomie élitiste véhiculé par le Michelin.

Cependant les nouveaux pays de gastronomie ont également entrepris de concurrencer la gastronomie française. La course à la créativité et à la sophistication est devenue européenne, voire mondiale quand New York et Tokyo sont devenues de nouvelles capitales gastronomiques. De plus, quand on arrive après les autres, on ne peut se faire de place qu'en se différenciant par la créativité, donc ici l'hyper-créativité.

Le cas de Ferran Adrià, proclamé meilleur cuisinier du monde, est emblématique de ces mutations. Quand il crée un œuf de caille en croûte de caramel, ou une polenta de parmesan, il n'est plus l'utilisateur de recettes ancestrales, mais un créateur - d'odeurs, de textures, de goûts. Avant de fermer définitivement son restaurant pour se consacrer à la création, il le fermait déjà pendant six mois et travaillait avec son équipe dans son "laboratoire" à Barcelone pour inventer de nouvelles recettes en utilisant de nouveaux procédés (cuisine à l'azote liquide, centrifugation, etc.) et des textures nouvelles (gélatines, sucettes, mousses ultra-aérées montées au siphon). Il exaltait la dimension sémiotique de sa cuisine en présentant ses plats comme des "tableaux" ; le dîner durait quatre heures et demie avec une succession, dans le cadre d'un menu unique, de trente tableaux. La créativité était mise en avant, Ferran Adria revendiquant 1500 créations estampillées, dont l'œuf de caille caramélisé, l'huître servie en écume d'eau de mer, le caviar servi sur une gelée de pomme, la gaufrette de parmesan surmontée d'une boule de glace au parmesan, ou les lamelles de champignon dégustées crues après avoir humé dans un carton leurs odeurs de sous-bois et ingéré une pipette de suc de cèpes. Cela lui a permis d'être considéré, y compris par nombre de chefs français, comme "le meilleur cuisinier sur la planète" (dixit Joël Robuchon). Les "consommateurs" l'ont également plébiscité : 400000 demandes de réservation pour 8000 places (le restaurant n'étant ouvert que le soir et pour 45 couverts).

\section{La dimension territoriale du système gastronomique français}

Le caractère scientifique et abstrait de la construction gastronomique française permettait, comme le faisait remarquer Parkhurst Ferguson, l'exportation de son modèle élitiste, mais impliquait en même temps une "déterritorialisation" de celui-ci en tant que processus culinaire. Cela implique-t-il pour autant que le système gastronomique français n'ait aucun rapport particulier au territoire, ce qui contredirait l'observation statistique de la concentration des restaurants dans certaines zones ? Absolument pas, car, pour traiter de sa dimension territoriale, il convient de tenir compte de la transformation du système gastronomique en système marchand et de l'approfondissement progressif de cette dimension marchande. Or celle-ci introduit une contrainte essentielle de rentabilité. Il ne suffit plus de "faire de la cuisine" pour les grands ou l'élite, il faut la vendre. Se pose donc la question nouvelle des débouchés et de leur inscription territoriale. La contrainte des débouchés est d'autant plus forte pour le système gastronomique français que celui-ci se fonde sur un modèle élitiste de cuisine coûteuse, dont la rentabilité marchande est problématique et dépend étroitement des débouchés. Il en est résulté que, loin de dépendre de la proximité de ressources spécifiques en termes d'inputs productifs, c'est la localisation de la clientèle qui a été le facteur dominant de localisation des restaurants en France. L'accroissement de la concurrence entre établissements a renforcé l'impératif de localisation à proximité des bassins de clientèle et l'a d'autant plus renforcé qu'elle entraînait une course au luxe synonyme d'investissements coûteux et donc de nouveaux problèmes de rentabilité. Et comme le processus culinaire élitiste était, comme nous l'avons dit, "déterritorialisé", c'est la contrainte de débouchés qui détermine la localisation des établisse- 
ments gastronomiques. Une partie de la gastronomie française a cependant noué des relations plus complexes avec son environnement en y intégrant la question de son approvisionnement.

La gastronomie parisienne a profité à plein de la centralisation française pour renforcer ses débouchés et assurer son expansion. Une continuité territoriale s'est ainsi affirmée entre gastronomie de cour, créée et développée autour des palais de la région parisienne, et gastronomie élitiste et marchande, qui s'appuie sur une clientèle concentrée dans la capitale. À la clientèle locale des élites financières et culturelles se joint l'importante clientèle des touristes qui ont fait de Paris la première destination touristique mondiale. L'accès à la gastronomie élitiste, régulièrement ou occasionnellement, des classes moyennes, et leur accès plus large aux segments inférieurs et moyens de la gastronomie qui ont "euphémisé" la cuisine de la niche élitiste (cf. supra) ont renforcé l'avantage de la gastronomie parisienne lié à la concentration de revenus médians et élevés dans la région parisienne, permis son expansion et reproduit sa domination. Paris, qui ne comptait que 3,6\% de la population française en 2010 , concentrait ainsi 38,5\% des troisétoiles Michelin, 15,5\% des deuxétoiles et 10,9\% des une-étoile.

Les cuisines élitistes situées hors de la région parisienne ont, elles aussi, été confrontées à l'épineuse question des débouchés. Une cuisine aussi coûteuse ne peut survivre qu'en pratiquant des prix élevés mais en réussissant néanmoins à obtenir des taux de remplissage suffisants. La localisation a donc suivi celle de la clientèle. L'échec de Pierre Gagnaire à Saint-Étienne, incapable d'y obtenir un équilibre financier, faute de "bassin de clientèle" suffisant, et son succès peu après à Paris, avec la même cuisine, est tout à fait symbolique. L'évolution de la localisation de la clientèle s'est traduite par l'expansion des restaurants de la Côte d'Azur et, plus largement, des zones à tourisme de luxe. La région Paca (Provence-Alpes-Côte d'Azur), avec 7,8 \% de la population française (chiffre 2010), concentre pour sa part 7,7 \% des trois-étoiles, $17,9 \%$ des deux-étoiles et 14,6\% des une-étoile ${ }^{(2)}$. Inversement, l'ancienne route des vacances que constituait la nationale 7 et le long de laquelle s'égrenaient les restaurants gastronomiques, dépouillée d'une partie de ses clients par l'expansion des autoroutes, a perdu de son lustre.

Quand la clientèle locale potentielle ne pouvait suffire à assurer l'équilibre financier des restaurants gastronomiques, il a donc fallu s'efforcer d'attirer de la clientèle, notamment touristique. Pour cela il convenait de justifier le détour ainsi occasionné. Il est d'ailleurs cocasse de constater que les références élitistes du Michelin, les deux et trois-étoiles qui s'adressent à la niche élitiste, se présentent précisément comme des justifications de détours (le deuxétoiles "mérite le détour" et le troisétoiles "mérite le voyage").

Attirer une clientèle qui ne se trouvait pas sur place et composer ainsi avec le principe dominant d'une localisation dictée par la clientèle impliquait alors de jouer sur les spécificités de la localisation réelle du restaurant. Ainsi les cuisines élitistes situées en région ont-elles été amenées à cultiver leur spécificité territoriale en jouant de leur proximité avec des actifs locaux spécifiques. Elles ont puisé dans les composantes non élitistes, bourgeoises ou populaires et d'origine régionale ou locale, du patrimoine gastronomique français. Avant que la clientèle à fort pouvoir d'achat n'ait massivement investi la Côte d'Azur, celle-ci a commencé à vanter la cuisine méditerranéenne, progressivement "redécouverte" voire "réinventée". La région lyonnaise, qui, sur la base d'une cuisine régionale affirmée, avait réussi à constituer un véritable district gastronomique (Bonnard, 20 I I), unissant cuisine élitiste et cuisine bourgeoise régionale, intégrant une cuisine résolument populaire quitte à la revisiter (les bouchons), a souvent représenté un modèle de solution. Néanmoins la contrainte de clientèle n'a pas permis d'obtenir une croissance aussi forte que celle qu'ont connue les régions, Paris en premier lieu, dotées de débouchés plus affirmés.

La contrainte de débouchés, particulièrement forte pour sa dominante élitiste, et son caractère de cuisine déterritorialisée ont a contrario stimulé l'expansion internationale de la cuisine française. Les plus grands chefs ont été amenés à s'investir sur les marchés gastrono- 
miques extérieurs en multipliant leurs implantations dans les grands bassins de demande gastronomique, les grandes métropoles mondiales, et en y réussissant grâce à leur réputation et à celle de la cuisine française. Obligés d'accorder leur cuisine traditionnelle avec les spécificités des goûts locaux, ils ont en même temps élargi leur palette de connaissances des goûts et saveurs, rencontré de nouveaux produits de qualité, et les ont intégrés dans leurs cuisines, y compris en France, accroissant par là même leur caractère déterritorialisé.

\section{Les effets sur}

\section{le développement territorial}

Il s'agit là d'une question difficile parce que les (rares) études qui l'ont affrontée sont surtout constituées de monographies, qui rendent difficile un jugement d'ensemble. Les statistiques disponibles sont ellesmêmes peu développées et de médiocre qualité. On ne pourra donc que se limiter à repérer les principaux types d'effets résultant du double processus que nous avons précédemment relevé : déterritorialisation du processus culinaire, territorialisation des débouchés via les bassins de clientèle.

- La course aux

\section{bassins de clientèle}

Nous avons vu plus haut comment la recherche de débouchés auprès d'une clientèle capable de consacrer des budgets accrus aux dépenses de restauration gastronomique, du fait de l'inflation de ses prix, conduisait à une restructuration spatiale de la cuisine élitiste. L'ensemble des deux régions Île-deFrance et Paca concentre aujourd'hui plus de $30 \%$ des restaurants étoilés, et le phénomène est encore plus marqué pour sa niche élitiste, plus de $46 \%$ des trois-étoiles et 34,5\% des deux-étoiles. Avec les inflexions de la localisation se modifient aussi les flux de revenus créés localement et les créations d'emplois.

La recherche de débouchés par la gastronomie la conduit à s'adresser aux touristes, nationaux mais surtout étrangers. Ce faisant, elle accroît aussi l'attractivité du territoire. Curnonsky cherchait, dès la fin de la Grande Guerre, à asseoir un cercle vertueux entre développement du tourisme et essor de la gastronomie, sur la base d'une valorisation du patrimoine culinaire français. La gastronomie française, qu'elle soit offerte par les restaurants de la niche élitiste, par ceux du luxe accessible ou par ceux qui s'inscrivent dans une gastronomie populaire et régionale, est un élément collectif important d'attrait touristique. Sa réputation est particulièrement consolidée par celle des grandes tables de la gastronomie élitiste, par effet d'image, même si la plupart des touristes n'y ont pas accès, mais elle est également soutenue par la réputation de l'ensemble des autres restaurants, de telle sorte que la France apparaît comme un, voire le "pays où l'on mange bien”. Cet effet concerne l'ensemble du territoire français et contribue à ce que la France apparaisse comme la première destination touristique au monde.
L'effet d'attirance globale se manifeste différemment selon les spécificités des territoires qui composent la nation, en fonction de l'importance des patrimoines gastronomiques locaux et de l'implantation des différents types d'établissements de restauration. Curnonsky visait déjà à faire de Lyon, et non de Paris, la capitale gastronomique du monde !

La niche élitiste constitue un point d'attraction essentiel. Elle s'adresse, compte tenu des prix pratiqués, à un segment très particulier de la demande gastronomique. Néanmoins ce segment, du fait de la mondialisation, du développement du tourisme international et de la diminution des coûts de transport, rassemble une clientèle quantitativement non négligeable et détentrice d'un pouvoir d'achat élevé qui participe aux revenus du tourisme. Ainsi, en dehors de l'effet général d'image dont bénéficie l'ensemble du territoire, cette niche élitiste produit des effets dérivés sur la consommation touristique, effets qui se concentrent avant tout sur Paris et, dans des conditions différentes, sur la Côte d'Azur. Elle contribue par là même à orienter le développement touristique. Les groupes financiers du luxe investissent dans les restaurants et hôtels. Les palaces parisiens, comme ceux de la Côte, recrutent des chefs de haut niveau pour gagner des étoiles au Michelin, asseoir leur réputation, accroître ou préserver leur clientèle de touristes. Les chefs eux-mêmes, confrontés au coût exorbitant de la cuisine élitiste, cher- 
chent à attirer les touristes et à rentabiliser leur cuisine par des prestations plus lucratives, adjoignent à leur restaurant de luxueuses chambres ou exercent directement leur talent dans les hôtels de luxe (comme propriétaires, copropriétaires ou simplement chefs). Dans certains cas la recherche de l'effet d'image susceptible d'attirer une clientèle ou de promouvoir l'image d'une région conduit les acteurs locaux, privés et publics, à soutenir les efforts des restaurateurs pour faire émerger des établissements de prestige, à réputation internationale. Les maisons de Champagne, par exemple, ont besoin que leurs clients, clients finaux ou négociants intermédiaires, puissent trouver en Champagne une restauration de qualité et développent des coopérations avec les établissements locaux haut de gamme. En outre, les effets sur le tourisme débordent du territoire national dans la mesure où la gastronomie élitiste française part à la recherche de nouveaux clients (locaux mais aussi touristes de passage) hors de son territoire d'origine, en ouvrant des restaurants dans toutes les grandes métropoles mondiales. Un autre effet dérivé est le développement des guides gastronomiques et particulièrement $\mathrm{du}$ Guide rouge (le "Michelin"), qui constitue un faire-valoir du modèle élitiste français.

Les restaurants de luxe accessibles et ceux de gastronomie populaire et régionale, offrant de la cuisine "bourgeoise" soignée, créent eux aussi des effets positifs pour le déve- loppement du tourisme. En particulier quand ils représentent une masse critique repérable, par leur agglomération dans certains territoires (la région lyonnaise ou le Périgord) ou le long de certains axes de communication, comme l'a été longtemps la nationale 7 .

L'expansion de la gastronomie française, portée par sa réputation nationale et internationale, par l'enrichissement d'une partie de la population mondiale et par le souci grandissant de satisfaction hédonistique, a des effets macro-économiques importants, en créant des emplois et en distribuant des revenus qui contribuent à soutenir la croissance. Son incidence sur le tourisme est également sensible en matière de balance des paiements. Il faut enfin noter que le modèle élitiste a permis le développement en France d'un secteur des arts de la table à forte réputation internationale, proposant des produits de grande qualité qui unissent une dimension artisanale ou industrielle à une forte dimension artistique (Baccarat, Lalique, Christofle...).

\section{- Des effets contrastés sur}

\section{la territorialisation des inputs}

Le modèle élitiste français est, on l'a vu, fondé sur un savoir scientifique et sur une compétence professionnelle et non sur la spécificité de ressources locales. La concentration de restaurants élitistes à Paris ou sur la Côte d'Azur n'a rien à voir avec l'origine locale des produits privilégiés par la "grande" cuisine française : lièvre, homard, huîtres, caviar, épices... La cuisine parisienne n'est pas issue d'un terroir agricole ou viticole, elle ne privilégie pas les asperges d'Argenteuil, le ginglet de Pontoise ou le vin de Montmartre. La cuisine élitiste s'approvisionne dans l'ensemble du territoire, voire à l'étranger. La concurrence entre établissements pousse à soigner la qualité des matières premières et la sélection des producteurs. Certains terroirs seront donc privilégiés mais leur localisation n'a rien à voir avec celle des établissements de restauration. Elle passe par l'interface des marchés et des réseaux d'intermédiaires, capables aujourd'hui de livrer n'importe quel produit en n'importe quel point du territoire. Le souci de qualité permet cependant à certains terroirs de survivre et à des productions artisanales de se maintenir en élevant la qualité de leur offre, et en contrant ainsi les fournisseurs qui standardisent leur production pour bénéficier d'économies d'échelle leur permettant de diminuer leurs prix.

Le modèle élitiste n'est pas un modèle économe. Il cuisine des produits rares dont il n'utilise que les parties ou composants nobles et gaspille ainsi des matières premières. Les faisant venir de tous les coins de France et du monde, il engendre des coûts de transport donc des coûts environnementaux élevés.

Les cuisines régionales qui ont développé des segments élitistes, comme la gastronomie lyonnaise ou bordelaise, cherchent de leur côté à préserver voire à développer des spécificités locales afin de pouvoir 
attirer localement une clientèle, et de justifier, notamment pour les touristes, de se détourner de la seule région parisienne : vins régionaux, poissons locaux (la lamproie, l'alose, l'omble chevalier), grenouilles des Dombes, charcuteries locales, asperges des Landes, chapons et canards du Sud-Ouest ou de Bresse, palombes... Les restaurateurs sont alors conduits à nouer des rapports qui dépassent le recours aux grands marchés d'intérêt national. Ils ont besoin d'un approvisionnement régulier et garanti. Ils incitent les fournisseurs à monter en qualité afin de pouvoir offrir, à leur tour, des prestations de qualité. En place de transactions marchandes standards et anonymes avec leurs fournisseurs, ils développent des relations marchandes personnalisées qui intègrent des exigences de qualité, de naturel, d'authenticité, sont fondées sur le voisinage et le partage de valeurs communes, et aboutissent à former des réseaux. Ainsi le restaurant mettra-t-il en valeur ses fournisseurs en publiant leurs noms ou en écoulant certains de leurs produits (des confitures, du miel, des moutardes, des foies gras, des objets de table...). Dominique Loiseau ${ }^{(3)}$ ou Olivier Roellinger multiplient les partenariats avec des producteurs locaux, qui les fournissent mais qu'ils peuvent aider à élever leur qualité ou à créer de nouveaux produits ou de nouvelles variétés grâce à leur expertise et à leur créativité. Ces relations peuvent aller jusqu'à des formes d'intégration quasi salariale. Laurent Tarridec, quand il travaillait aux Mouscardins, deuxétoiles à Saint-Tropez, devant la difficulté à obtenir un approvisionnement local régulier, avait décidé de salarier l'un des pêcheurs du port. Ces cuisines élitistes régionales jouent ainsi la carte du retour au terroir et des circuits courts, s'efforçant d'utiliser leur localisation et leur spécialisation culinaire pour surfer sur la vague des nouvelles valeurs de culte de la nature et de respect de l'environnement. Cependant le culte du naturel, dans le contexte de la montée des valeurs écologiques, conduit l'ensemble du secteur à limiter et à contrôler son recours au marché. Ainsi plusieurs grands chefs parisiens suivent-ils l'exemple emblématique d'Alain Passard, en créant leurs propres potagers pour aboutir à une quasiintégration de certains approvisionnements.

Les restaurants gastronomiques de gamme inférieure, qui développent des offres inspirées des cuisines populaires et régionales, jouent dans un contexte concurrentiel tendu par la montée des coûts. Ils sont donc pris entre deux tendances contradictoires. D'un côté, s'adresser aux grands réseaux commerciaux d'approvisionnement pour bénéficier de la standardisation et des baisses de prix. De l'autre, privilégier leurs spécificités locales et leur connaissance des réseaux de producteurs de qualité pour, comme les restaurants du segment supérieur, valoriser la "typicité" de leurs prestations par l'utilisation de "produits authentiques" et la constitution de réseaux personnels de petits fournisseurs. Il en est également ainsi pour les vins avec la recherche systématique du "petit producteur", censé apporter un produit authentique et sain et avoir été découvert par le chef, ce qui, compte tenu de la concurrence et du nombre croissant de restaurants, lui donne un caractère de plus en plus mythique. Ce mouvement s'accélère aujourd'hui en s'appuyant sur la demande croissante de "vrai", de "naturel", de "proche", formulée par les consommateurs ou qui leur est attribuée.

Les restaurants gastronomiques représentent évidemment un débouché direct pour les producteurs vitivinicoles tout en contribuant à asseoir la réputation de leurs vins et alcools. Comme les restaurants attirent une partie de leur clientèle par la qualité de leur offre en ce domaine, des synergies existent entre gastronomie et production vitivinicole. Les restaurants, y compris ceux de la niche élitiste, privilégient donc les produits locaux et présentent des cartes incluant de très nombreux noms et variétés (Les Crayères, à Reims, proposent quatre cents références de champagnes). Inversement, les syndicats de producteurs vitivinicoles et les grands producteurs, comme cela est ou a été le cas pour les maisons et les grandes coopératives en Champagne et pour les propriétaires de grands crus dans le Bordelais, s'efforcent de favoriser la constitution d'une offre gastronomique de qualité (en relayant les politiques de communication des chefs ou en leur consentant des 
conditions d'achat particulièrement favorables), susceptible de compléter l'attrait que représente la qualité de leurs vins, afin d'attirer une clientèle qui achètera leurs produits, en parlera et contribuera ainsi à consolider leur réputation et à élargir leurs débouchés.

Cette analyse exploratoire montre que l'essentiel des effets de l'expansion gastronomique tend à se diluer sur l'ensemble du territoire, du fait du principe de non-territorialisation de la cuisine scientifique et artistique propre au modèle élitiste. En même temps, des inflexions dans ce phénomène sont à remarquer. Se pose alors la question de leur avenir : pourraient-elles modifier la liaison gastronomie-développement territorial, et cela d'autant que le modèle élitiste semble donner des signes de faiblesse?

\section{UN ÉPUISEMENT DES EFFETS D'ENTRAÎNEMENT DU MODÈLE ÉLITISTE ?}

De nombreux éléments conduisent, depuis le début des années 1990, à une remise en cause du modèle gastronomique élitiste. Ils dépassent le cas de la France dans la mesure où le modèle dominant de gastronomie dans le monde était fondé sur le modèle français et faisait que, quand une gastronomie émergeait, elle s'efforçait de reprendre les modes d'organisation et les valeurs de ce modèle.

La dynamique globale de la gastronomie française est restée marquée jusqu'à présent par la préémi- nence de son modèle élitiste. Or celui-ci rencontre aujourd'hui des difficultés nouvelles. Si certains grands restaurants français sont rentables, s'exportent dans le monde entier (les treize restaurants de Joël Robuchon totalisent dix-huit étoiles Michelin, le dix-neuf de Paul Bocuse, dix-sept), d'autres rencontrent de grandes difficultés à trouver un équilibre financier. De plus, un nombre croissant de restaurants gastronomiques renoncent à une cuisine de type élitiste pour "renouer avec les racines de la gastronomie”. Une telle évolution, apparemment liée à la "démocratisation" de la gastronomie, questionne l'hégémonie du vieux modèle de gastronomie élitiste. Si de nombreux chefs sont toujours engagés dans une course aux étoiles, au luxe et à la sophistication, d'autres, comme Alain Senderens (qui a pendant vingt-huit ans affiché les trois étoiles de son Lucas Carton, place de la Madeleine à Paris), renoncent volontairement à leurs étoiles et recherchent un nouveau type de prestations en expliquant vouloir désormais appliquer "les principes $d u$ low cost à une activité de luxe, la gastronomie" (Senderens, 2007).

\section{Les difficultés du business model du grand restaurant}

Nombre de chefs réputés ont ces dernières années adopté une attitude iconoclaste, parce que contraire à toute la tradition existant jusque là, en renonçant volontairement à leurs étoiles Michelin. Alain Senderens (Lucas Carton, Paris, Île-de-France),
Olivier Roellinger (Les Maisons de Bricourt, Cancale, Bretagne), Philippe Gaertner (Aux Armes de France, Ammerschwihr, Alsace), Hervé Paulus (Hostellerie Paulus, Landser, Alsace) en France mais aussi Ezio Santin (Antica Osteria del Ponte, Cassinetta di Lugagnano, Lombardie) ou Gualtiero Marchesi (Gualtiero Marchesi Restaurant A L'Albereta, Erbusco, Lombardie) en Italie, tous deux détenteurs de trois étoiles Michelin, sont les figures les plus éminentes de ce mouvement.

Une première raison expliquant l'inversion du mouvement d'inflation de luxe qui avait précédemment caractérisé la gastronomie française vient de la hausse continue des coûts exigés par la compétition élitiste. Michel Guérard (trois étoiles, à Eugénie-les-Bains) signale ainsi que le prix d'un fauteuil dans sa salle de restaurant atteint aujourd'hui 1000 euros pièce ; au Meurice de Yannick Alleno, les chandeliers individuels Lalique coûtent 700 euros pièce. À cela s'ajoute l'effet Baumol bien connu (Baumol et Bowen, 1966) : dans des secteurs avec un processus de production fondé sur le travail vivant, les gains en productivité sont très limités (à l'inverse des secteurs industriels), de sorte que le prix relatif des biens des premiers ne peut qu'augmenter de façon continue. Dès lors la gastronomie élitiste est condamnée à la hausse des prix. Les données du Guide rouge montrent, sur la période 1950-2010, que plus la cuisine est élitiste, plus la hausse des prix a été forte et que la hausse s'accélère après 2000. Les grands 
restaurants, pris entre le coût de matières premières toujours plus chères et des frais salariaux croissants, sont condamnés à proposer des additions de plus en plus élevées mais doivent aussi afficher des taux de remplissage élevés. Or les prix fous risquent de décourager la clientèle de masse, sauf si fonctionne un effet superstar : le menu "Soir d'hiver” à L'Arpège, d'Alain Passard, est à 420 euros, le "blanc de bar braisé et glacé au champagne" du Meurice est facturé à la carte 142 euros. Le grand restaurant élitiste ne peut alors se maintenir que dans des lieux où passe une clientèle riche, internationale, se renouvelant sans cesse. La niche élitiste ne disparaît donc pas mais se contracte et ne constitue plus "Le" modèle de la gastronomie. En outre, ces établissements, de plus en plus adossés à des hôtels et à des groupes de luxe, pour lesquels ils constituent aussi une vitrine créatrice d'image et de réputation, obéissent à une polarisation géographique qui s'accélère (Paris, Lyon, la Côte d'Azur, les stations de sports d'hiver haut de gamme...) ; ailleurs, l'on trouve des trois-étoiles avec des salles à moitié vides et nombre d'entre eux doivent se reconvertir. Marc Veyrat a dû vendre son trois-étoiles La Ferme de mon père, à Megève, en janvier 2007, L'Espérance de Marc Meneau est depuis plusieurs années en situation instable, après avoir été mise en redressement judiciaire, et nous avons déjà parlé du parcours de Pierre Gagnaire.

Une seconde raison explique les difficultés du modèle de restaurant élitiste. Il s'agit de la question de la succession du créateur. Le principal capital du restaurant élitiste est en effet la réputation de son chef, directement liée à son talent et à son investissement personnel dans l'établissement. Il est donc de type idiosyncrasique, dépendant de la personne même du chef créateur. Quand le chef disparaît rien n'assure automatiquement le maintien de la valeur du capital qu'il portait. Que Marc Veyrat soit un grand cuisinier n'implique pas nécessairement que son successeur, beaucoup moins connu, soit aussi un grand cuisinier et que sa prestation justifie une addition astronomique. Quand la succession est assurée par un fils (les dynasties Troisgros ou Bras) ou une fille (Pic à Valence), les clients potentiels peuvent anticiper une continuité qui existe beaucoup moins quand la succession ne garantit aucune continuité particulière ( $c f$. les difficultés d'un restaurant comme Les Crayères à Reims, où plusieurs chefs réputés se succèdent sans arriver à retrouver la gloire d'antan après le retrait de Gérard Boyer qui avait lui-même succédé à son père, Gaston). C'est pourquoi, outre le recours à la succession familiale, les restaurants élitistes s'efforcent de passer, comme dans le domaine de la haute couture (Barrère et Santagata, 2005), de la "griffe" (le nom propre du créateur) à la "marque" (une garantie de qualité), ce qu'ont réussi par exemple Robuchon, Bocuse ou Ducasse. Dès lors que chacun possède plus de dix restaurants dans le monde et multiplie les activités annexes, tout le monde sait qu'ils ne sont plus derrière les fourneaux dans chacun de leurs établissements mais leur nom est gage de qualité : un restaurant Bocuse est un restaurant dans lequel le chef aura été sélectionné avec soin, les procédures de qualité seront respectées, les approvisionnements se feront auprès de producteurs de qualité... et ainsi de suite.

\section{Les changements dans la demande}

Avec l'accroissement des niveaux de vie des classes moyennes dans les pays industrialisés s'élargit la clientèle de la gastronomie. Le développement de la consommation de biens offrant des caractéristiques hédonistes, révélé entre autres par l'inflation du discours gastronomique dans les médias, y contribue également. Ce faisant, la demande gastronomique ne s'inscrit plus seulement dans une logique élitiste, telle que celle qui justifiait l'hégémonie du grand restaurant. La sophistication est remise en cause par nombre de nouveaux consommateurs et par les critiques gastronomiques qui reflètent leurs goûts et préférences. De nouveaux chefs partagent ces convictions et proposent de nouvelles cuisines. Quand Ferran Adrià se distingue par la sophistication exacerbée de sa cuisine, d'autres privilégient, en les traitant avec plus ou moins de créativité, les produits naturels. Ainsi, les légumes, habituellement considérés en France comme nourriture com- 
mune, deviennent l'élément central des recettes d'Alain Passard, qui, dans son trois-étoiles parisien, présente la viande ou le poisson comme accompagnement des légumes, érigés au rang de cœur du plat.

La mise en valeur des produits naturels, l'insistance sur le lien entre produits ou recettes et territoires, leur conception comme éléments de patrimoines nationaux, régionaux ou locaux, répondent à des demandes d'identité des consommateurs. La volonté de respecter les saisons, de s'approvisionner auprès de producteurs de qualité et de petite taille, respectueux des savoir-faire traditionnels, de favoriser la proximité répond à la montée des valeurs écologiques. L'ouverture à de nouvelles saveurs, à de nouvelles façons de travailler les produits correspond à la progression du multiculturalisme, du désir d'entrer en contact avec de nouveaux patrimoines, plus ou moins éloignés de sa culture d'origine. Le chef est de moins en moins reconnu pour son aptitude à présenter ou travailler des produits de luxe (caviar, langouste, ris de veau, truffes...) et de plus en plus pour sa créativité.

Cependant, la tradition aristocratique qui fondait la cuisine élitiste française et lui donnait un avantage comparatif indiscutable freine son adaptation aux changements du monde et à la circulation des cultures, y compris culinaires. Il institutionnalise des formes définies d'offre (le menu avec sa déclinaison de plats valorisant les produits rares et de qualité, destiné à offrir de l'ex- tra-ordinaire), des modes de composition et de préparation (les sauces et apprêts, la transformation du produit...). Tout cela crée des barrières au changement et rend plus difficile l'intégration d'éléments d'autres cultures culinaires. Des phénomènes de lock-in se développent, au moment où émergent de nouvelles offres culinaires, dans de nouveaux pays gastronomiques (l'Espagne, voire l'Angleterre ou les pays nordiques), moins prisonniers de patrimoines rigides, plus flexibles et capables de s'adapter plus rapidement.

\section{La confusion créée par la cuisine industrialisée}

Les mutations sociales intervenues dans la phase de croissance de l'après-guerre conduisent à une augmentation du nombre des repas pris à l'extérieur du domicile ainsi qu'à la recherche d'économie de temps dans la préparation des repas appartenant à la sphère domestique. Les industries agro-alimentaires trouvent là de puissantes incitations à rechercher des innovations économes en temps. Surgelés, fours à micro-ondes, nouvelles techniques de conservation envahissent cuisines domestiques et professionnelles. La restauration commerciale, pour lutter contre l'effet Baumol, adopte massivement ces nouvelles techniques et externalise une part considérable et croissante du processus de production culinaire. La cuisine s'industrialise. Ce mouvement touche aussi le domaine gastronomique de la restauration commerciale. Pour les segments supé- rieurs, la brigade reste l'élément décisif du processus de production et les technologies sont utilisées pour améliorer la qualité : le four à vapeur sèche permet des cuissons plus précises et plus courtes qui respectent mieux les propriétés des matières premières et exaltent les saveurs.

Cependant l'industrialisation de la cuisine permet à des établissements de "singer" à moindres frais les caractéristiques élitistes de la gastronomie traditionnelle, en se procurant à bon prix des produits jadis de luxe et désormais livrés "clefs en main" par les nouvelles chaînes de l'agro-alimentaire ou en économisant sur les frais de préparation. Dans nombre de restaurants prétendument gastronomiques, de niveau moyen, les cuisiniers se transforment en assembleurs et réchauffeurs de produits industrialisés arrivant tout prêts, notamment grâce aux nouvelles techniques de conservation (dont la cuisson sous vide). Des économies fortes en coûts de travail sont alors possibles, grâce à la réduction des temps de préparation des plats et à la substitution de main d'œuvre peu qualifiée aux employés hautement qualifiés du passé. Les grandes firmes de l'agro-alimentaire, qui ont d'abord proposé ces produits aux ménages, développent désormais des lignes professionnelles qui s'adressent à la restauration commerciale. À côté des chaînes de restauration industrielle de masse de type McDonald's et Pizza Hut, une partie croissante de la restauration commerciale traditionnelle, y compris des établissements jusque là 
considérés comme gastronomiques, bascule désormais dans une nouvelle catégorie, celle de la restauration industrialisée (en France, l'évolution du groupe Flo est tout à fait éclairante). L'industrialisation des goûts alimentaires participe du mouvement de marchandisation de la culture, lui-même lié à la possibilité de standardiser et reproduire en série les biens et à l'exigence de rentabiliser les nouveaux capitaux investis dans ces domaines, mouvement que dénonçait déjà avant guerre Walter Benjamin.

La gastronomie se trouve interpellée par ces mutations. Elle l'est d'autant plus que son marché est caractérisé par une information très imparfaite qui rend difficile la distinction entre "vraie" et "fausse" gastronomie et complexifie le choix des consommateurs potentiels. Des réactions interviennent donc qui peuvent modifier les relations entre système gastronomique, dynamique gastronomique et dynamiques territoriales.

\section{Vers un pluralisme gastronomique?}

Une partie de la cuisine élitiste se maintient dans cette niche quand ses conditions de marché (localisation adaptée, appui sur un parc immobilier de chambres, recettes annexes liées à l'exploitation de la réputation, présence d'un cuisinier superstar, présence dans les médias...) le permettent. Une autre, notamment quand elle possède moins d'atouts compétitifs spécifiques (Senderens n'exploitait pas de chambres à côté de son restaurant), se lance dans une gastronomie de luxe épurée dans laquelle est censée perdurer la qualité gastronomique de la cuisine, mais qui économise sur le décor et remplace les produits très coûteux par des produits qui le sont moins. Le pari peut s'avérer gagnant quand la réputation du chef, souvent constituée lors de sa période élitiste, assure une clientèle nombreuse ou que le boucheà-oreille fonctionne. La gageure de cette nouvelle gastronomie est d'économiser sur le décor et les produits de luxe sans diminuer la qualité de la cuisine proprement dite, ce qu'exprime Senderens en précisant que sa cuisine, elle, est restée trois étoiles. Elle conduit aussi, pour justifier le remplacement de produits "nobles" par des produits plus courants, à soigner l'origine des produits en nouant des relations plus étroites avec des fournisseurs sélectionnés, toutes choses qui entrent en résonance avec la montée et des valeurs écologiques et des demandes d'identité, du "retour aux racines", de la mise en valeur des patrimoines. En même temps cela permet à cette nouvelle restauration gastronomique de marquer sa différence avec la restauration industrialisée des "réchauffeurs" de plats. La pression à l'innovation, et en premier lieu à l'innovation dans l'approvisionnement, touche également le rapport au vin. Pour rester dans des prix raisonnables, il n'est plus possible d'organiser le repas autour de premiers crus dont les prix, sous l'influence de la forte hausse de la demande mondiale alimentée par les nouvelles classes aisées des pays émergents, atteignent aujourd'hui des montants astronomiques. D'où la recherche de petits producteurs de vins plus naturels, de nouvelles formes d'association entre plats et vins et de consommation de vin (les vins au verre, les ensembles verre de vin-plat...).

Ainsi, le modèle élitiste n'est-il plus l'unique modèle d'organisation de la gastronomie. Une sorte de pluralisme gastronomique se développe avec l'apparition de nouveaux modèles de restauration gastronomique. Les façons très sophistiquées de cuisiner ne sont plus la seule incarnation du luxe et du goût. La créativité appliquée à des matières premières traditionnelles attire une clientèle de plus en plus large, à la recherche de nouveaux codes alimentaires, attentive à la qualité et à la salubrité des produits, et prenant ses distances avec le luxe et le conformisme du cadre traditionnel du grand restaurant. "Gastronomie low cost", nouvelle gastronomie urbaine mais surtout gastronomie locale et populaire (au besoin, elle aussi, "revisitée") contribuent à la démocratisation et à la consommation de masse de la gastronomie. Si dans un premier temps la démocratisation de la gastronomie a été seulement "formelle", c'est-à-dire que l'accès en a été ouvert à un plus large public mais qu'elle est restée organisée selon le modèle élitiste, lui-même issu du modèle aristocratique, désormais la démocratisation tend à devenir "réelle" en jouant 
aussi sur son contenu et en s'écartant des codes d'inspiration aristocratique. Cela renforce les chances des cuisines locales qui recourent à des patrimoines locaux et cela inaugure de nouveaux liens entre développement gastronomique et développement local des territoires.

Émerge en deuxième lieu un nouvel ensemble de gastronomie de qualité, autour de jeunes chefs innovants, souvent distingués dans le Guide rouge par l'attribution des "Bib”. Il s'agit généralement de restaurants nouveaux ou d'anciens établissements repris et profondément transformés, plus nécessairement situés dans les zones traditionnelles de gastronomie (les beaux arrondissements parisiens ou lyonnais). Ils innovent en s'inspirant du patrimoine gastronomique français mais en s'inspirant aussi des cultures culinaires et gastronomiques étrangères. Plus flexibles que les grands établissements, ils peuvent s'adapter plus rapidement aux modes, à l'évolution des valeurs (respect de l'environnement, recherche de nourritures saines et légères, utilisation d'épices...) et, notamment, à la recherche de produits typiques, inscrits dans des cultures locales. Leur émergence est facilitée par la mutation observée avec l'irruption d'internet dans la critique gastronomique. Le développement des forums de discussion et blogs gastronomiques accélère succès et échecs tandis que la nouvelle gastronomie "démocratisée", en tout cas "décomplexée", trouve un débouché dans des communautés de gastronomes (parfois caricaturés comme "bobos") dans lesquelles l'information circule très rapidement. Leur pérennité ne repose pas seulement sur la qualité de leur cuisine et de leurs innovations de produit mais aussi sur celle de leur gestion et des innovations introduites dans celle-ci pour limiter les coûts tout en permettant le renouvellement régulier de l'offre et la surprise du consommateur. Cette "nouvelle scène de la gastronomie française", qui cherche à tisser un lien plus étroit au territoire (quitte à mélanger produits locaux et saveurs venues d'ailleurs) reste cependant limitée aux grandes villes et aux lieux de passage de nombreux touristes (Côte d'Azur, Sud-Ouest, stations de montagne) dans lesquels la clientèle potentielle est nombreuse.

Enfin, des gastronomies locales qui s'étaient vues peu ou prou niées ou absorbées dans le modèle élitiste parisien développent leurs spécificités, mettent en avant la typicité de leurs produits locaux et s'émancipent de la tutelle élitiste pour renouer avec une cuisine populaire et partagée. Une partie de la niche élitiste qui se maintient dans ces régions s'aligne sur ces valeurs, voire, comme dans le cas emblématique de Michel Bras, cherche à les transcender.

\section{CONCLUSION :}

LE PLURALISME GASTRONOMIQUE, UNE CHANCE POUR

LE DÉVELOPPEMENT LOCAL ?

Dans nombre de pays, il existe une gastronomie populaire, généralement fondée sur les produits du terroir et les recettes locales. Cela est d'autant plus vrai en Europe que la France s'était singularisée par son extrême centralisation alors que les autres nations conservaient de fortes autonomies régionales. Il en est ainsi en Espagne avec les cuisines catalane, andalouse ou basque, dont l'ancrage territorial, contre le pouvoir centralisateur madrilène, conduit à des symbioses fortes entre cuisine, culture régionale et participation "citoyenne" (cf. Etcheverria, 2008, sur les associations gastronomiques de San Sebastian). En Italie, les cuisines locales ont toujours existé, qu'elles aient gardé une forme rurale ou qu'elles aient été sauvegardées à la ville dans le cadre urbain via les cours princières ou le mouvement communal. Elles ont conservé une partie de leurs singularités, comme en Sicile ou en Sardaigne, en Piémont ou en Toscane, à Venise ou à Bologne.

Les stratégies récentes de valorisation des patrimoines gastronomiques locaux et régionaux dans ces pays et les innovations organisationnelles mises en œuvre peuvent être à l'origine d'un modèle gastronomique nouveau et alternatif, fondé sur une dynamique locale très particulière, profondément différente de celle qu'a jusqu'ici enclenchée le modèle dominant dans la gastronomie mondiale. Les mouvements italiens Slow Food ou Kilometro zero (qui examine les bilans carbone des matières premières utilisées par les restaurants gastronomiques et privilégie l'approvisionnement local), tout comme 
les nouvelles entreprises de l'agroalimentaire (Eataly, par exemple) qui valorisent les productions locales à forte typicité et sont capables de les exporter sur le marché mondial, poussent à l'instauration de ce nouveau modèle gastronomique. Celui-ci s'appuierait sur une relation au territoire local beaucoup plus forte que dans le cas du modèle élitiste et capable, dans certains cas, de constituer, sur le modèle des districts culturels (Santagata, 2006), de véritables districts gastronomiques : ils disposent en effet de ressources spécifiques (produits locaux, savoir-faire locaux, recettes locales, associations locales gastronomiques et culturelles), souvent conservées et transmises par des patrimoines locaux, ressources spécifiques sur la base desquelles la proximité culturelle peut développer des synergies entre ressources et entre acteurs (Bonnard, 20II). Ces districts gastronomiques bénéficient des économies d'agglomération et de "colocalisation" et, plus largement, de la création d'une "atmosphère" culturelle (au sens d'Alfred Marshall, 1890) favorable à l'innovation en gastronomie. Ils ne se contentent pas de promouvoir des produits locaux ou de retourner à des traditions anciennes. Ils présentent au contraire un nouveau visage, moderniste, de l'alimentation, de la gastronomie et de leur insertion dans une culture sociétale. Ils multiplient les innovations, en particulier organisationnelles, se montrent capables de flexibilité et d'initiative pour saisir les oppor- tunités de marché. Le réseau Eataly introduit de la nouveauté dans la gestion logistique comme dans le marketing. Le mouvement Slow Food constitue désormais un réseau de masse, avec ses institutions et ses formes propres d'organisation, ses liens avec d'autres pays (on sait qu'il a essaimé avec succès aux États-Unis).

En même temps ces districts et ces organisations se révèlent beaucoup plus aptes que le modèle gastronomique élitiste à tisser des liens dynamiques avec les territoires. Le rapport développement gastronomique-développement local est tout à fait différent et beaucoup plus fort. Le développement de formes associées aux gastronomies locales comme l'agrotourisme et l'œnotourisme en témoigne clairement. Alors qu'en 2002 les routes des vins n'étaient que seize seulement en France, porteuse d'un vignoble a priori plus réputé, on en comptait déjà quatre-vingt-dix-huit en Italie. L’hétérogénéité des liens entre alimentation et territoire - de la forme dominante en Piémont qui associe avant tout gastronomie et culture, voire art, à celle qui l'emporte en Toscane où est privilégiée la relation entre cuisine et promotion des produits locaux - montre aussi que ces cuisines s'inscrivent dans des patrimoines locaux dont le contenu contribue à les modeler.

Le système gastronomique français saura-t-il résister à cette nouvelle concurrence, pourra-t-il, compte tenu du poids historique de son patrimoine, s'adapter aux mutations du monde gastronomique et inventer, à son tour, de nouveaux rapports au territoire ? C'est tout l'enjeu des années à venir.

\footnotetext{
NOTES

(*) L'auteur remercie les deux referees dont les remarques ont permis d'améliorer la cohérence et la clarté du texte. Selon la formule consacrée, il reste seul responsable des insuffisances qui peuvent persister.

(I) Elle fait ainsi partie des "hedonic products", biens à utilité essentiellement
} hédoniste que Addis et Holbrook (200I) distinguent des "utilitarians products", biens à utilité essentiellement utilitaire, et des "balanced products", dont l'utilité combine les deux types définis.

(2) La localisation par effet de clientèle est merveilleusement illustrée par la dernière édition du Guide rouge (20।3), qui ne consacre qu'un seul nouveau trois-étoiles, le restaurant La Vague d'or, partie d'un complexe hôtelier cinq étoiles situé à... SaintTropez.

(3) Elle affirme que $55 \%$ des matières premières utilisées dans son restaurant phare sont issues de Bourgogne. 


\section{RÉFÉRENCES BIBLIOGRAPHIQUES}

Michela AdDIs et Morris B. HOLBROOK, "On the conceptual link between mass customisation and experiential consumption: an explosion of subjectivity, Joumal of Consumer Behaviour, vol. I, n I, 200 I, pp. 50-66. Christian Barrère, Denis Barthélemy, Martino Nieddu et Franck-

Dominique Vivien, Réinventer le patrimoine. De la culture à l'économie, une nouvelle pensée du patrimoine?, L'Harmattan, 2004.

Christian BARRÈre et Walter SANTAgATA, La mode. Une économie de la créativité et du patrimoine, à l'heure du marché, La Documentation française, 2005.

Christian BARRÈre, Quentin BONNARD et Véronique ChOSSAT,

"Democratization in the gastronomic market: from Michelin stars to Michelin 'Bibs"', The $16^{\text {th }}$ ACEI Conference, Copenhague, 9-12 juin 2010. Christian BARRÈre, Aldo BuZIO, Alessia MARIOTtI, Alessandro CorSI et Paola BORRIONE, "Industria del gusto : un nuovo paradigma italiano", Florens 2012. Essays and Researches. International Biennial of Cultural and Environmental Heritage, Bandecchi \& Vivaldi, 2012.

William J. Baumol et William G. Bowen, Performing Arts - the Economic Dilemma: A Study of Problems Common to Theater, Opera, Music and Dance, The MIT Press, 1966.

Bénédict Beaugé, "Sur l'idée de nouveauté en cuisine", Mode de recherche, n 13 ("Gastronomie, cycles de mode et consommation"), 2010.

Quentin BONNARD, Les districts gastronomiques, des districts d'un nouveau type, thèse de doctorat es sciences économiques, Université de Reims Champagne-Ardenne, 2011.

Jean Anthelme Brillat-Savarin, Physiologie du goût, Gabriel de Gonet, 1848 (paru en 1826).

Olivier ETCHEVERRIA, "San Sebastian : capitale gastronomique parmi les capitales gastronomiques", dans Julia CSERGo et Jean-Pierre LEMASSON (dir.), Voyages en gastronomies. L'invention des capitales et des régions gourmandes, Autrement, 2008.

Olivier ETCHeVERriA, "Introduction", Norois, nº 219 (numéro spécial dirigé par Olivier ETCHEVERRIA : "Le restaurant, outil de développement local ?"), Presses universitaires de Rennes, septembre 20 I I, 20 I la.

Olivier EtCheverRIA, "Les tensions fondatrices du développement local autour du restaurant Bras à Laguiole : 'centralité', qualité et créativité", Norois, n² 219 (numéro special dirigé par Olivier ETCHEVERRIA : “Le restaurant, outil de développement local ?'), Presses universitaires de Rennes, septembre 201 I, 20 I Ib.
Claude FISCHLER, L'Homnivore. Le goût, la cuisine et le corps, coll. "Points", Odile Jacob, 1990.

\section{Elsa Gatelier, Marie Delaplace et Marie-Claude Pichery,}

Patrimonialisation de la vitiviniculture et développement du tourisme dans les régions viticoles. Une comparaison Bourgogne/ Champagne, XLIXe colloque de l'ASRDLF, Belfort, 9-II juillet 2012.

Sylvie GUICHARD-ANGUIS, "Diffusion d'une culture alimentaire régionale et restauration : Kii Tanabe (Japon)", Norois, n²19 (numéro special dirigé par Olivier ETCHEVERRIA : “Le restaurant, outil de développement local ?'), Presses universitaires de Rennes, septembre 201 I.

Gilles LIPOVETSKY, L'Empire de l'éphémère. La mode et son destin dans les sociétés modernes, Gallimard, 1987.

Vincent MARCILHAC, "Revitalisation d'une économie locale et stratégie familiale : le cas Marcon à Saint-Bonnet-le-Froid", Norois, n² 219 (numéro special dirigé par Olivier ETCHEVERRIA : “Le restaurant, outil de développement local ?'), Presses universitaires de Rennes, septembre 20। I.

Alfred Marshall, Principles of Economics, $8^{e}$ edition, Macmillan, 1920 (paru en 1890).

Priscilla Parkhurst Ferguson, Accounting for taste. The triumph of French cuisine, The University of Chicago Press, 2004.

Jean-Pierre PoulaIN, Manger aujourd'hui. Attitudes, normes et pratiques, Privat, 2002.

Walter SANTAGATA, "Cultural districts and their role in developed and developing countries", dans Victor A. GINSBURGH et David THROSBY (dir.), Handbook of the Economics of Art and Culture, North-Holland, 2006.

Alain Senderens, "La créativité sans les étoiles. De Lucas Carton à Senderens", Le Journal de l'école de Paris du management, n 63, 2007.

Georg Simmel, "Fashion", The American Journal of Sociology, vol. 62, n 6 , 1957 (article paru en 1904)

Herbert SPENCER, "On Manners and Fashion" (paru en 1854 dans Westminster Review), dans H. SPENCER, Essays on Education and Kindred Subjects, Dent/Everyman, réédition 1966 (Ireédition 191 I). Thorstein VeBLEN, The Theory of the Leisure Class, MacMillan, 1899. 